\title{
Changes in Corneal Collagen Architecture during Mouse Postnatal Development
}

\author{
Jack Sheppard,${ }^{1,2}$ Sally Hayes,,${ }^{1,2}$ Craig Boote, ${ }^{1}$ Marcela Votruba, ${ }^{1}$ and Keith M. Meek ${ }^{1}$
}

Purpose. To characterize changes in corneal collagen arrangement during mouse postnatal development.

Methods. Small-angle X-ray scatter patterns were gathered from the centers of 32 excised mice corneas aged between postnatal days 10 (before eye opening) and 28 (onset of sexual maturity). These were analyzed to produce measurements of the average separation distance between corneal collagen fibrils. Changes in the predominant orientation of corneal collagen and its relative distribution during the same developmental period were determined using wide-angle X-ray scatter data collected at $0.2-\mathrm{mm}$ intervals over the entire cornea and limbal region of each specimen.

Results. Collagen interfibrillar spacing decreased in the days leading up to eye opening $(61.3 \pm 2.9 \mathrm{~nm}$ at day 10 to $45.5 \pm$ $4.5 \mathrm{~nm}$ at day 14), after which it remained constant. However, changes in collagen orientation and distribution occurred throughout the entire developmental period. After eye opening at day 12, collagen alignment gradually increased in the peripheral cornea and limbus. By day 28, an annulus of highly aligned collagen surrounded the cornea.

Conclusions. Changes in corneal thickness before and after eye opening are not caused by widespread alterations in the collagen fibrillar array but are more likely caused by expansion and contraction of regions devoid of regularly arranged collagen. The postnatal development of a corneal annulus of collagen, thought to play a role in stabilizing the curvature of the cornea, may be triggered by visual factors. (Invest Ophthalmol Vis Sci. 2010;51:2936-2942) DOI:10.1167/iovs.09-4612

$\mathrm{T}$ he cornea is the transparent window at the front of the eye and acts as its main refractive component. Mouse models are invaluable tools in the study of both normal corneal development and the development of corneal dystrophies. ${ }^{1-3}$ Their accelerated lifespan ( 1 mouse year $\approx 30$ human years) and rapid generation time provide an ideal opportunity to identify early-stage changes that occur in a particular pathogenesis before clinically diagnostic symptoms become apparent. However, to establish a baseline from which gene-targeted mutations and disease-induced changes can be distinguished, it is

From the ${ }^{1}$ School of Optometry and Vision Sciences, Cardiff University, Cardiff, United Kingdom.

${ }^{2}$ These authors contributed equally to the work presented here and should therefore be regarded as equivalent authors.

Supported by UK Medical Research Council Programme Grant G0600755 (KMM), an MRC studentship, and a CCLRC Beam-time Programme Grant (KMM). KMM is a Royal Society-Wolfson Research Merit Award Holder.

Submitted for publication September 10, 2009; revised November 30, 2009; accepted January 8, 2010.

Disclosure: J. Sheppard, None; S. Hayes, None; C. Boote, None; M. Votruba, None; K.M. Meek, None

Corresponding author: Keith M. Meek, School of Optometry and Vision Sciences, Cardiff University, Maindy Road, Cardiff CF24 4LU, United Kingdom; meekkm@cf.ac.uk. necessary to first characterize the ultrastructure of the mouse cornea during normal postnatal development.

Eye opening in the mouse occurs at 12 to 14 days postpartum $^{4}$ and is preceded by a series of well-documented changes in the cornea that prepare it for interactions with the outer environment. These include an increased proliferation of epithelial cell layers, a thickening of the stromal layer, a decrease in stromal density, and a reduction in light scattering. ${ }^{5}$ Corneal transparency is largely dependent on the diameters of stromal collagen fibrils and their precise organization within the tissue. ${ }^{6,7}$ The arrangement of corneal collagen is such that uniformly narrow fibrils lie parallel to each other in layers (lamellae), which are themselves organized in an ordered, latticelike configuration. 8,9 This unique arrangement of collagen results in the destructive interference of light scattered away from the forward direction. Thus, all the light energy goes into the constructive interference in the forward direction, resulting in corneal transparency.

The precise orientation of stromal collagen, which varies with corneal position and differs between species, is thought to play an important role in the biomechanics of the tissue by resisting the forces exerted on the cornea to maintain correct corneal curvature. ${ }^{10-12} \mathrm{X}$-ray scattering studies have shown that an annulus of highly aligned collagen surrounding the cornea at the limbus is a common feature in primates ${ }^{10,11}$ and other mammals, including the mouse. ${ }^{2,12}$ It is thought that this feature may play a role in maintaining the curvature of the cornea at the point where it meets the lesser curved sclera. ${ }^{10,11}$

To characterize the early structural development of the mouse cornea between postnatal day 10 (before eye opening) and the onset of sexual maturity at day $28^{13}$ and to establish at what stage in development an annulus of collagen is formed around the cornea, a series of detailed X-ray scattering studies was conducted using synchrotron radiation. The data were analyzed to produce quantitative information about the average separation distance between collagen fibrils, their predominant orientation, and their relative mass distribution in the corneas of mice at different stages of postnatal development.

\section{Methods ANd Materials}

\section{Tissue Collection and Preparation}

In accordance with the ARVO Statement for the Use of Animals in Ophthalmic and Vision Research, 48 whole eyes were obtained from wild-type 129/S1 laboratory mice (Jackson Laboratories, Bar Harbor, ME) aged 10, 11, 12, 13, 14, 15, 21, and 28 days (six eyes at each time point). The cornea from each eye was excised, and its precise in vivo orientation was marked by means of a surgical suture. Immediately after dissection, each cornea was wrapped in plastic wrap (Clingfilm; Superdrug Stores, Croyden, UK) to prevent tissue dehydration and was transferred to $-80^{\circ} \mathrm{C}$ storage until required for X-ray data collection. Although freezing is known to reduce the diameters of corneal collagen fibrils and their separation distance, it has been shown that both these parameters return to normal when the tissue is thawed. ${ }^{14}$ 

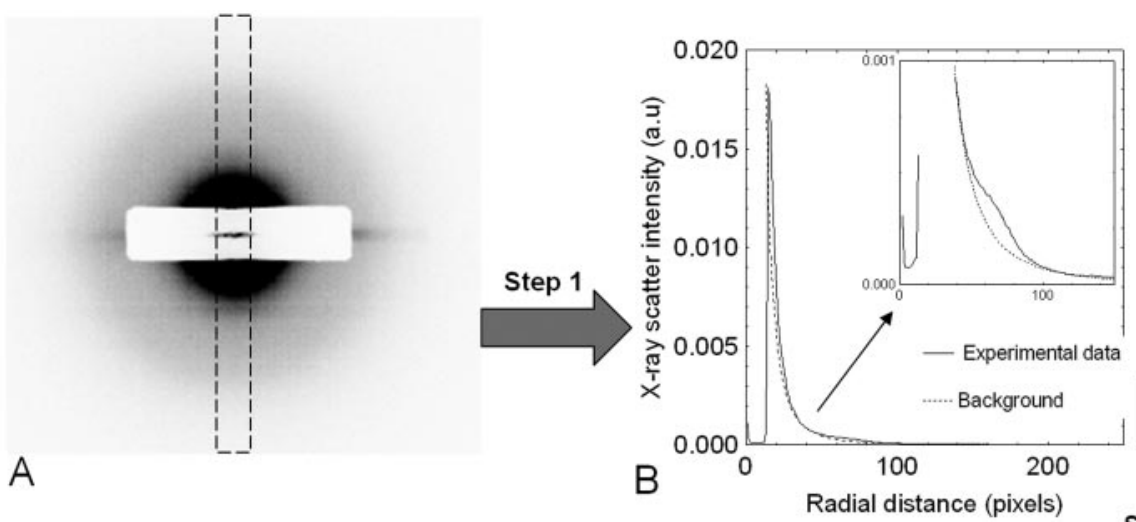

FigURE 1. Analysis of small-angle $X$ ray scattering data. In step 1 , a vertical transect (dashed line) was taken through the center of the pattern (A), and the resultant intensity profile folded (B). A square-power law background was then fitted to the experimental data (B) and subtracted. The profile was divided point-for-point by the fibril transform (Bessel function) (C) to yield the interference function (D), the peak position of which is determined by the interfibrillar Bragg spacing of collagen.
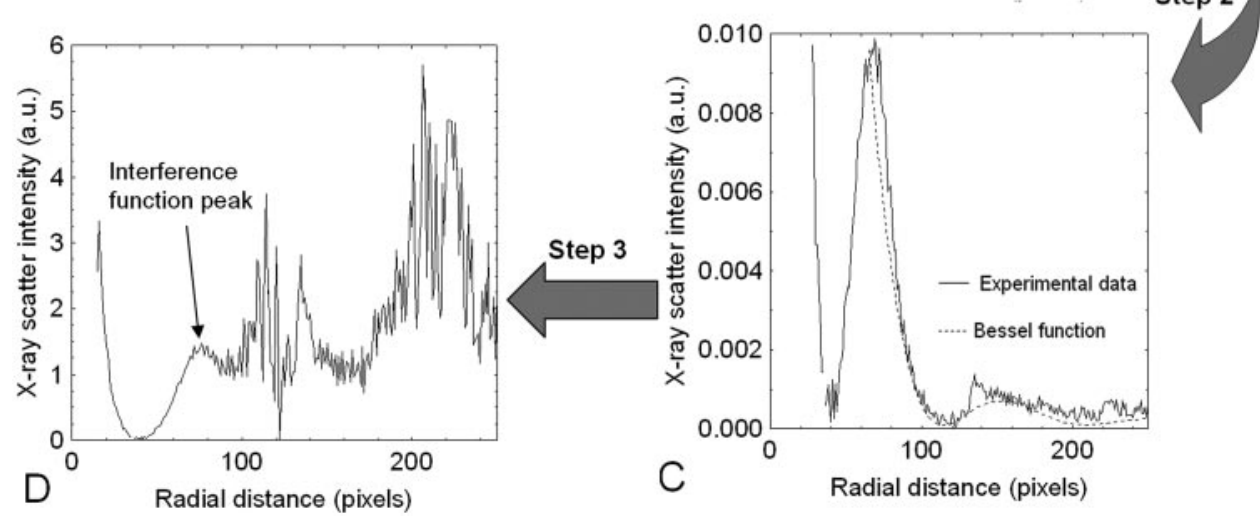

\section{Small-Angle X-Ray Scattering Data Collection and Analysis}

Small-angle X-ray scattering patterns were obtained from all corneas $(n=48)$ on Station 2.1 at the UK Synchrotron Radiation Source (Daresbury, UK) using a 9-m-long camera and an X-ray beam with a wavelength of $0.154 \mathrm{~nm}$ and a cross-sectional area at the specimen of $0.5 \mathrm{~mm} \times 1 \mathrm{~mm}$ (vertical $\times$ horizontal). To minimize tissue dehydration during X-ray exposure, each cornea (in plastic wrap) was placed in a sealed polymethyl methacrylate (Perspex; theplasticshop.co.uk, Coventry, UK) chamber with polyester film (Mylar; DuPont-Teijin, Middlesbrough, UK) windows. Although sufficient space was provided between the front and the back surfaces of the chamber to prevent corneal compression and distortion, each cornea was examined microscopically before data collection to confirm the absence of any folds in the tissue. For 3 minutes, X-rays were passed through the center of each cornea (parallel to the optical axis), and the resultant X-ray scatter pattern (Fig. 1A) was recorded on a multiwire gas detector. Measurements of corneal weight ( 4 decimal places) obtained before and after $\mathrm{X}$-ray exposure showed there to be no significant change in corneal hydration during data collection.

The X-ray scatter patterns were analyzed as described previously by Boote et al. ${ }^{15}$ using Unix-based software and statistics and graphics packages (Fit2D [European Synchrotron Radiation Facility, Grenoble, France]; Excel [Microsoft Corp., Redmond, WA]; Statistica [Statsoft, Tulsa, OK]) to produce measurements of the average center-to-center collagen fibril Bragg spacing.

A vertical transect was taken through the center of each X-ray scatter pattern (Fig. 1A) to generate an intensity profile of the firstorder equatorial (i.e., collagen interfibrillar) X-ray reflection. This was then folded about the center to improve the signal-to-noise ratio (Fig. 1B). To remove the background scatter from nonfibrillar tissue components, a square-power law background curve was fitted and subsequently subtracted from the intensity profile (Fig. 1B).

To obtain the interference function, which is derived from the ordered arrangement of fibrils and represents the probability of finding a fibril center at a given distance from another fibril center, a theoret- ical fibril transform (Bessel function) was fitted to the first subsidiary maximum of the experimental data (Fig. 1C), and the data were divided point for point by the calculated fibril transform. The resultant interference function (Fig. 1D), the peak position of which is determined by the average interfibrillar Bragg spacing, was then calibrated against the position of the first-order X-ray reflection and known 67-nm D-periodicity of hydrated rat tail tendon.

Comparisons between developmental days in terms of collagen interfibrillar spacing were statistically evaluated using ANOVA. This was followed by a separation of means test to identify between which groups significant differences occurred.

\section{Wide-Angle X-Ray Scattering Data Collection and Analysis}

Wide-angle X-ray scattering data from four corneas at each time point $(10,11,12,13,14,15,21$, and 28 days) were collected on Station 14.1 at the UK Synchrotron Radiation Source using a $200 \mu \mathrm{m} \times 200 \mu \mathrm{m}$ $\mathrm{X}$-ray beam with a wavelength $0.1488 \mathrm{~nm}$.

The tissue was mounted as for small-angle X-ray scattering data collection (in plastic wrap [Clingfilm] and placed in an airtight polymethyl methacrylate [Perspex; Databank] chamber with polyester film [Mylar; DuPont-Teijin] windows). The specimen chamber was then positioned so that the X-ray beam was directed toward the anterior surface of the cornea.

Using a specimen translation stage (Newport Ltd., Newbury, UK) interfaced with the X-ray camera shutter and a charge-coupled device detector (Quantum 4R; ADSC, Poway, CA) located $150 \mathrm{~mm}$ behind the specimen, X-ray scatter patterns-each resulting from a 60-second exposure-were gathered at $0.2-\mathrm{mm}$ intervals in all directions across each mouse cornea. X-ray scatter patterns, such as those shown in Figure $2 \mathrm{~A}$, are formed by the interference of X-rays scattered by regularly arranged collagen molecules lying near-axially within the stromal fibrils. ${ }^{16}$ If lamellae are orientated equally in all directions throughout the thickness of the cornea, a circular X-ray scatter pattern of uniform intensity is formed. However, if there is an excess of lamellae lying in a particular direction within the cornea (preferentially 

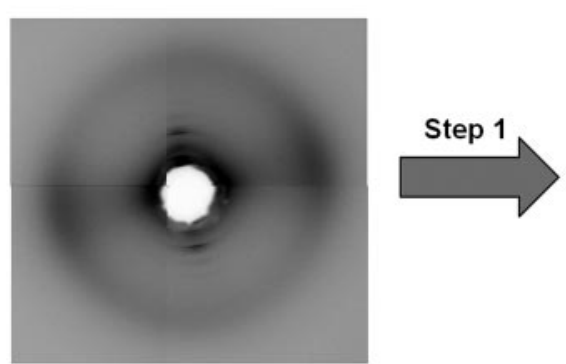

A

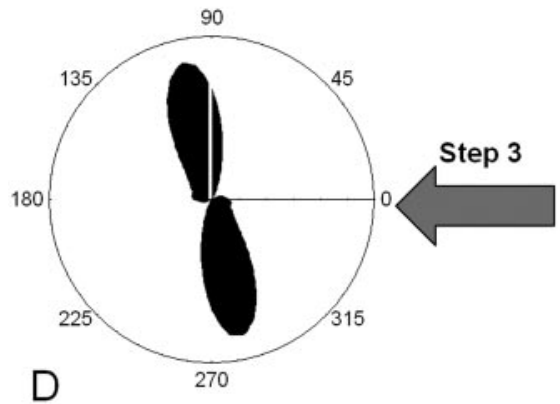

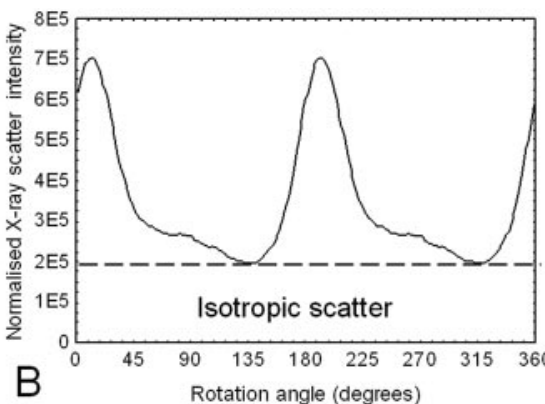
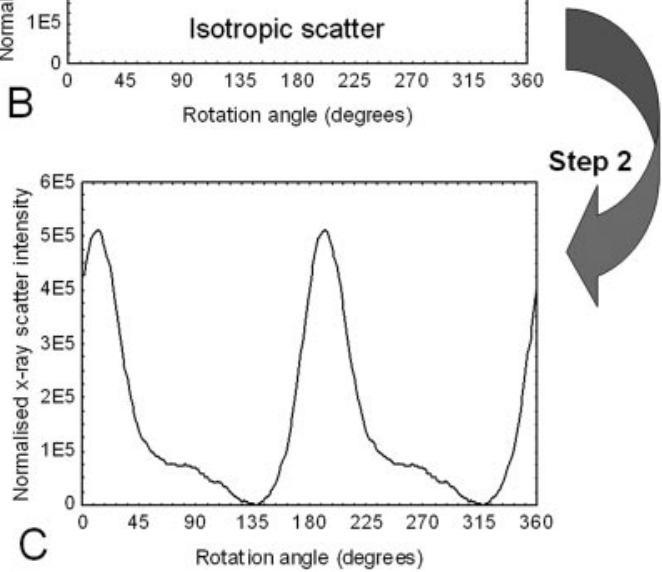

FIGURE 2. Major steps of wide-angle $\mathrm{X}$-ray scatter pattern analysis. An Xray scattering pattern from the limbus (A) shows a ring of X-ray scatter from collagen lying in all directions in the plane of the tissue (isotropic scatter) and two lobes of increased scatter intensity from collagen that are preferentially aligned. The angular distribution of scattered X-ray intensity is measured (B), and the scatter from isotropically arranged collagen removed to leave just the scatter from preferentially aligned collagen (C). This profile is then shifted by $90^{\circ}$ and converted to a vector plot (D) in which the radial distance represents the amount of collagen aligned in that direction. aligned collagen), the resultant X-ray scatter patterns show lobes of increased X-ray scatter (Fig. 2A) at right angles to the predominant direction of the collagen lamellae.

Each wide-angle X-ray scattering pattern was analyzed as described by Abahussin et al. ${ }^{17}$ using Unix (Fit2D)- and Windows (Microsoft Excel, London, UK)-based software and an image analysis package (Optimas 6.5; Media Cybernetics, Bethesda, MD) to obtain measurements of (1) the total X-ray scatter and, hence, the relative collagen mass density and (2) the angular distribution of X-ray scatter (predominant orientation of collagen) averaged through the thickness of the cornea at each sampling position. X-ray scatter patterns were first normalized against the intensity of the beam at the time of data collection. The center of the X-ray scatter pattern was then located, and the intensity of scatter around the circumference of 90 concentric circles (spanning from the center of the pattern to just beyond the ring of X-ray scatter) was measured. The circumference of each concentric circle was then subdivided into 256 sectors, and the average intensity in each sector was recorded. Background caused by flare from the backstop and scattering from nonfibrillar tissue components was removed by fitting a power law curve to either side of the collagen peak on the plot of scattering intensity versus radial distance and subsequently subtracting it. ${ }^{18}$ This was performed for all 256 radial intensity profiles to account for uneven flare from the backstop and changes in background with radial direction. Each profile was then radially integrated to give a single intensity value at each point on the circumference of the collagen reflection. The profile of collagen X-ray scatter relative to radial position $(0-256)$ was then converted to a profile of collagen X-ray scatter relative to angular position $(0-360)$ around the $\mathrm{X}$-ray pattern (Fig. 2B). The total area under the graph at this stage is proportional to the amount of collagen and is composed of scatter from collagen lying in all directions (isotropic scatter) and scatter from preferentially aligned collagen. Uniform background scatter from isotropic collagen was removed to produce a profile of scatter from preferentially aligned collagen only (Fig. 2C). The intensity profile was then shifted by $90^{\circ}$ to account for the fact that X-rays are scattered at right angles to the direction of the fibril axis, thereby showing the amount of aligned collagen as a function of the actual angle at which they occur in the tissue. By plotting these data as a $360^{\circ}$ vector plot (Fig. 2D), the distance from the center of the plot in any given direction represents the amount of fibrils preferentially oriented in that direction at that point in the tissue, based on an average measurement throughout the entire thickness of the cornea. Individual vector plots were arranged according to their geometric position on the cornea to produce vector plot maps showing the predominant orientation of collagen across each cornea.

In addition, contour maps showing the relative distribution of total collagen and preferentially aligned collagen throughout each cornea were formed by assembling calculated values of X-ray scatter intensity relative to corneal position.

\section{RESUlts}

\section{Collagen Fibril Spacing}

In the days leading up to eye opening, the average center-tocenter spacing of collagen decreased significantly from 61.3 $\mathrm{nm} \pm 2.9$ at day 10 to $45.5 \mathrm{~nm} \pm 4.5$ at day $14(P=0.002 ;$ Fig. 3 ). After eye opening (days 14-28) a nonsignificant increase in interfibrillar spacing was observed (45.5 $\mathrm{nm} \pm 4.5$ to 49.2 $\mathrm{nm} \pm 4.7)$. Developmental changes in interfibrillar spacing are not statistically related (based on linear regression analysis) to the transient variations in stromal thickness before and after eye opening, as reported by Song et al. ${ }^{5}$ (Fig. 3).

\section{Relative Distribution of Collagen}

Figure 4 shows the relative distribution of aligned collagen in typical right corneas from mice between 10 and 28 days of age. In the central cornea, preferentially aligned collagen mass increased substantially between postnatal days 10 and 11, after which only small localized fluctuations were detected. An increase in aligned collagen was observed in the peripheral region of the cornea at each developmental stage. Between days 10 and 12, an increase in the intensity of X-ray scatter from preferentially aligned collagen was evident in two opposing quadrants of the peripheral cornea (superior-nasal and inferior-temporal), indicating a relative increase in aligned collagen in these regions. The intensity of X-ray scatter from preferentially aligned collagen continued to increase throughout development in these two quadrants, and from day 13 onward aligned collagen also increased, though to a lesser extent, in the remaining regions of the peripheral cornea. By day 28 of postnatal development, a near complete annulus of highly aligned collagen surrounded the cornea. 
FIGURE 3. The relationship between collagen interfibrillar Bragg spacing (solid line, square markers) and stromal thickness (broken line, circular markers) in the developing mouse cornea. Values for stromal thickness, based on measurements cited in the text (days 12, 21, and 30) and calculated estimates from the graphics display (days 10 and 14) were obtained from Song et al. ${ }^{5}$ Error bars represent SD.

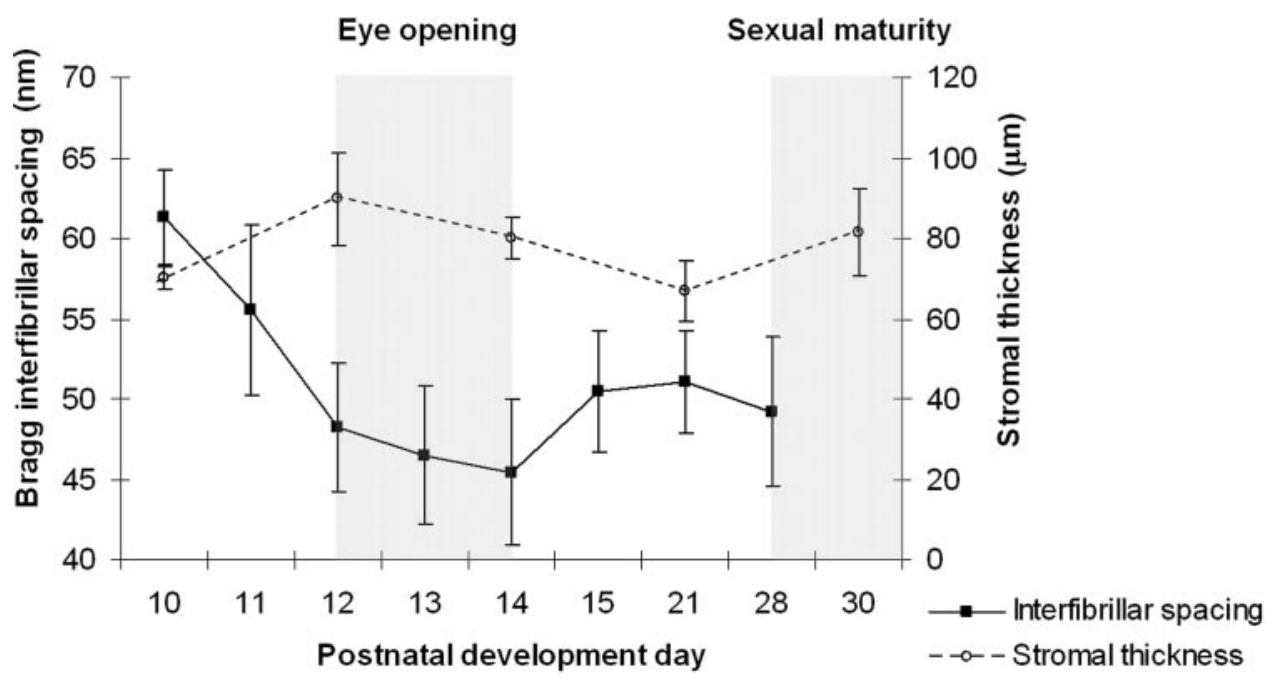

\section{Predominant Collagen Orientation}

Figure 5 shows the predominant orientation of corneal collagen in the developing mouse (right cornea shown in each case). Although there is no consistent preferred orientation of collagen in the very center of the cornea, from day 11 onward there is a tendency for collagen to lie predominantly in a horizontal (15/28 corneas examined) or oblique (superior temporal to inferior nasal in 13/28) direction. Beyond this central region, collagen adopts a tangential orientation with respect to the edge of the cornea. During development, the amount of tangentially aligned collagen gradually increases in the peripheral region of the cornea (as shown by the need for higher

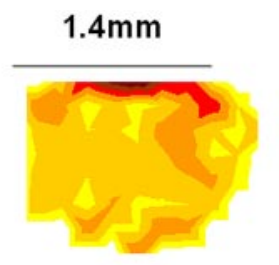

\section{D10}

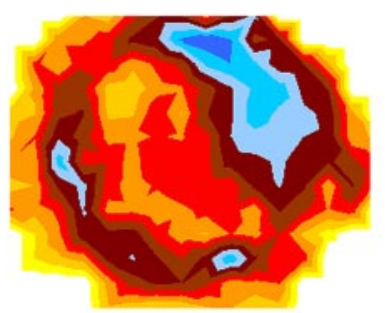

D13

Figure 4. Contour maps showing changes in the relative distribution of aligned collagen in the mouse cornea before and after eye opening. Measurements of X-ray scatter intensity (au) were made at $0.2-\mathrm{mm}$ intervals across the corneas of mice aged between 10 and 28 days (D) of postnatal development. A typical right eye is shown in each case.

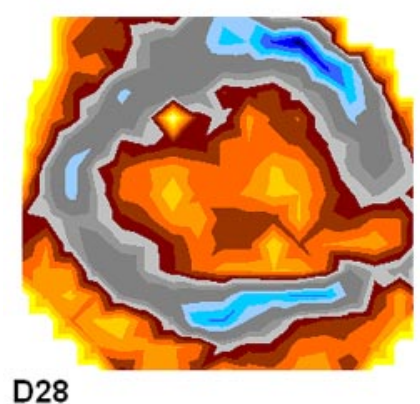

vector plot scaling factors), resulting in the formation of an annulus of highly aligned collagen by day 28 .

\section{Discussion}

This detailed investigation of mouse corneal development between the ages of 10 and 28 days substantiates the trends observed by Beecher et al. ${ }^{19}$ in a lower resolution X-ray scattering study of collagen interfibrillar spacing between the ages of 8 and 14 days. However, measurements of collagen parameters at corresponding developmental time points vary between the two studies, likely reflecting differences in the
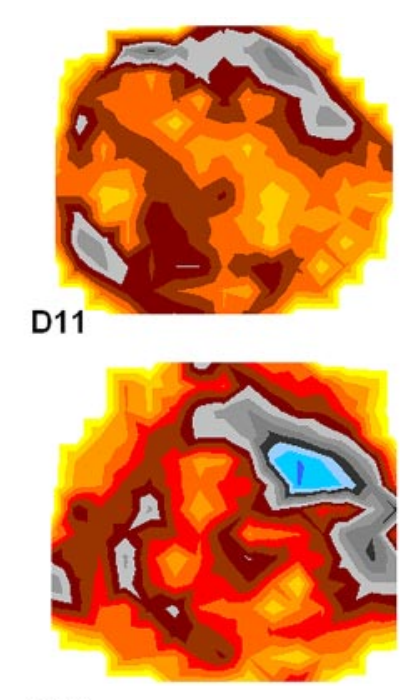

D14

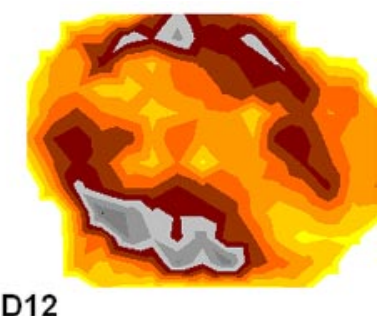

D12

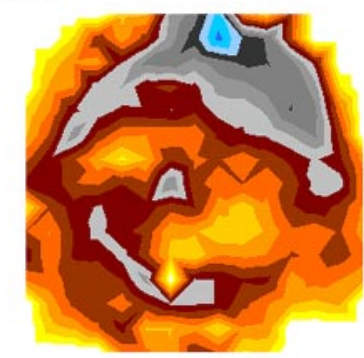

D15

\begin{tabular}{|ll|}
\hline $0-1$ & $\square-2$ \\
$2-3$ & $\square-4$ \\
$\square-5$ & $\square 5-6$ \\
$\square 6-7$ & $\square-8$ \\
$\square 8-9$ & $\square 9-10$ \\
$\square 10-11$ & $\square 11-12$ \\
$\square 12-13$ & $\square 13-14$ \\
$\square 14-15$ & $\square 15-16$ \\
\hline
\end{tabular}




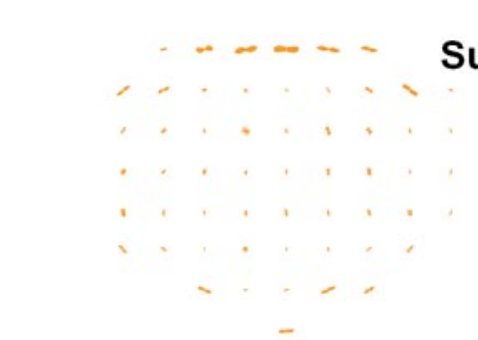

D10

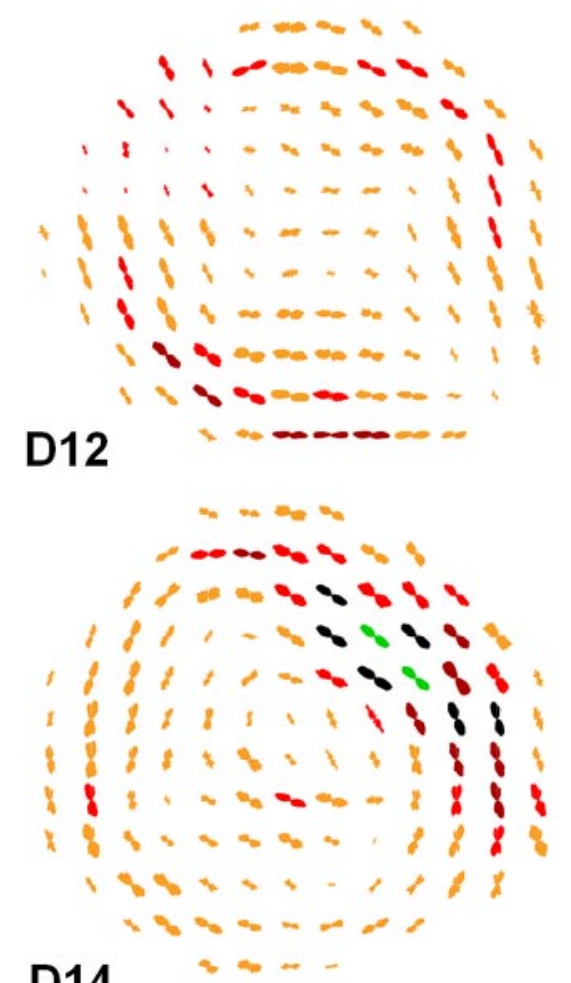

D14

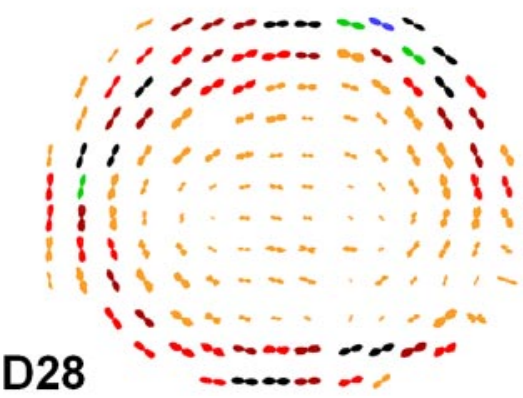

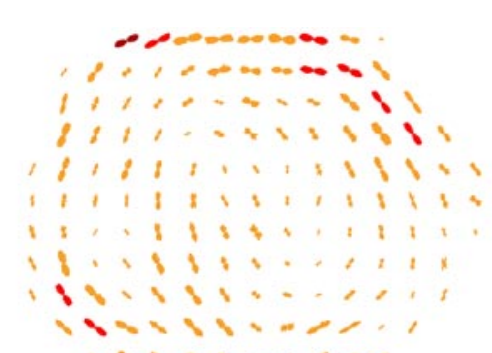

D11
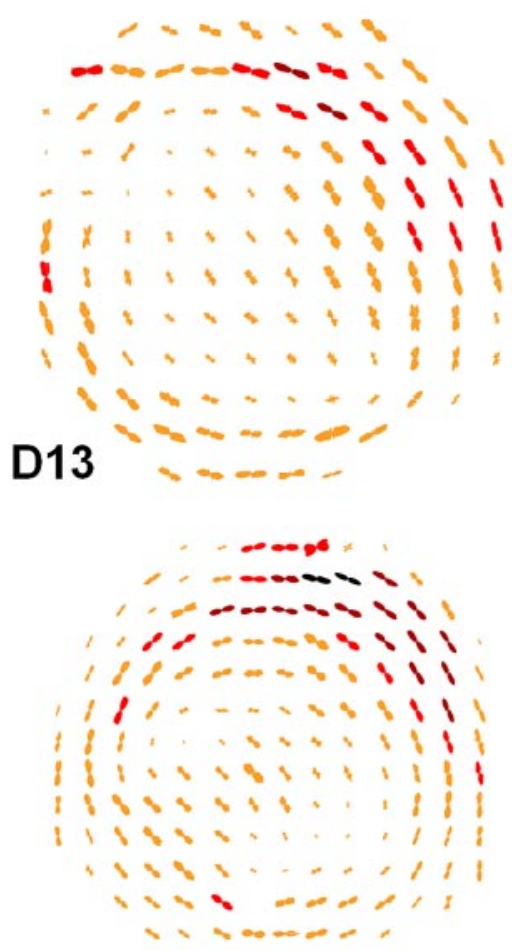

D15

\section{Scaling factors}

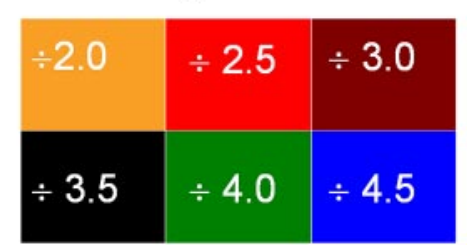

Figure 5. Vector plot maps showing changes in the predominant orientation of collagen in the mouse cornea pre and post eye-opening. Data were collected at $0.2-\mathrm{mm}$ intervals across the corneas of mice aged between 10 and 28 days (D) of postnatal development. A typical right eye (same as shown in Fig. 4) is shown in each case. For montage display it was necessary to scale down some of the vector plots (by the factors shown in the color key). Regions of high collagen alignment (such as at the limbus) required higher scaling down factors than regions of lower collagen alignment (central cornea). At day 10, the polar plots are barely visible due to the small amount of collagen alignment at this stage. sample preparation technique (freezing vs. chemical fixation) because all other aspects of the methodology were the same. Given that it has been shown that collagen interfibrillar spacing returns to normal physiological values on thawing, ${ }^{14}$ the measurements presented in this study can be considered to closely represent those of fresh tissue.

As shown here, stromal swelling in the mouse cornea before eye opening is not accompanied by an increase in collagen interfibrillar spacing, and the subsequent thinning of the stroma that occurs immediately after eye opening ${ }^{5}$ does not coincide with a compaction of collagen fibrils. Hence, the findings presented in this article support the belief of Beecher et al. ${ }^{19}$ that transient changes in stromal thickness before and after eye opening are not caused by widespread changes in the fibrillar array but are more likely the result of the expansion and contraction of regions of the stroma devoid of regularly arranged collagen.

In addition to transient changes in the thickness of the mouse corneal stroma and the proximity of constituent collagen fibrils ${ }^{5}$ during postnatal development, our results also show that changes in collagen orientation and distribution occur between postnatal days 10 and 28, thus implying that collagen reorganization and deposition are ongoing processes. The major changes seen in the relative distribution of collagen in the limbal region are of particular note. Previous work has shown that an annulus of highly aligned collagen circumscrib- 
ing the cornea at the limbus is a common structural feature among primates, ${ }^{10,11}$ other mammals, ${ }^{12}$ and chickens. ${ }^{20}$ Although its precise function is unknown, it is thought that it may provide the additional strength needed to reinforce the cornea at the point where it meets the less curved sclera. Maurice $^{21}$ estimated that, in humans, the change in curvature would produce in a circumferential tension at the limbus of at least twice that of neighboring regions. Indeed, its mechanical role in corneal curvature stabilization is supported by evidence of corneal annulus disruption in adult mice with a murinespecific keratopathy in which males are prone to corneal ectasia $^{2}$ and the absence of a corneal annulus in chickens with an inherited recessive condition (retinopathy globe enlarged) that is characterized by globe enlargement and flattening of the cornea. ${ }^{20}$ However, one question remains: if the limbal annulus of collagen is responsible for the maintenance of corneal curvature, how does the curvature of the cornea change during emmetropization? In most mammalian species, the eye is hyperopic at birth and gradually becomes emmetropic over time as a result of compensatory changes in axial length and the curvatures of both the cornea and the lens. ${ }^{22-27}$ In humans, for example, it has been shown that corneal curvature varies throughout the first year of life,$^{28}$ but the changes are most pronounced during the initial 2 to 4 weeks of infancy. ${ }^{29}$ We believe the answer to this question lies in the fact that, in the mouse at least, an annulus of collagen circumscribing the cornea is not present at birth but is formed postnatally between the period of eye opening and sexual maturity. Based on numerous studies showing the importance of visual experience in the control of eye growth and the development of refractive state (see Ref. 30 for review), we propose, first, that the development of the mouse corneal annulus after eye opening provides evidence that visual factors are involved in its development and, second, that its absence at birth may facilitate corneal shape change during a period of rapid infantile eye growth (between birth and day 22 of development). ${ }^{25}$ Its subsequent formation at day 28 may help to prevent any further corneal shape changes from taking place once the growth of the eye has stabilized. ${ }^{25}$ However, because of discrepancies (likely caused by variations in mouse strains and the technical difficulty associated with obtaining keratometry readings from corneas measuring approximately $2-3 \mathrm{~mm}$ in diameter) between studies regarding the age at which the curvature of the mouse cornea becomes stable, ${ }^{25,31}$ it is clear that further investigations are needed to confirm the precise relationship between the cessation of corneal shape change and the formation of a limbal annulus of collagen.

In conclusion, the findings presented here suggest that changes in corneal thickness before and after eye opening are not caused by large-scale alterations in the organization of collagen fibrils but are more likely caused by expansion and contraction of regions devoid of regularly arranged collagen. Furthermore, the postnatal development of an annulus of highly aligned collagen surrounding the cornea, which is initiated at eye opening and ends when the curvature of the cornea begins to stabilize, strengthens the belief that this structure plays a major role in maintaining the shape of the cornea. It is, therefore, conceivable that abnormalities in the development or maintenance of this structural feature may be at least partially responsible for pathologic corneal shape changes that occur during adolescence, such as seen in the human disease keratoconus.

\section{Acknowledgments}

The authors thank Mike McDonald (Station 14.1), Gunter Grossman (Station 2.1), and the staff at the UK Synchrotron Radiation Source for their assistance in data collection.

\section{References}

1. Pal-Ghosh S, Pajoohesh-Ganji A, Brown M, Stepp MA. A mouse model for the study of recurrent corneal epithelial erosions: alpha 9 beta 1 integrin implicated in progression of the disease. Invest Ophthalmol Vis Sci. 2004;45:1775-1788.

2. Quantock AJ, Dennis S, Adachi W, et al. An annulus of collagen fibrils in the mouse cornea and alterations in the form of hereditary keratoconus. Invest Ophthalmol Vis Sci. 2003;44:1906-1911.

3. Smith RS, Hawes NL, Kuhlmann SD, et al. Corn1: a mouse model for corneal surface disease and neovascularization. Invest Ophthalmol Vis Sci. 1996;37:397-404.

4. Smith RS, Kao WW-Y, John SWM. Ocular development. In: Smith RS, John SMW, Nishina PM, Sundberg JP, eds. Systematic Evaluation of the Mouse Eye. Washington: CRC Press; 2002:57.

5. Song J, Lee YG, Houston J, et al. Neonatal corneal stromal development in the normal and lumican-deficient mouse. Invest $O p h$ thalmol Vis Sci. 2003;44:548-557.

6. Farrell R, McCally R. Corneal transparency. In: Albert DM, Frederick A, Jakobiec, MD, eds. Principles and Practice of Ophthalmology. Philadelphia: WB Saunders; 2000;629-643.

7. Meek KM, Leonard DW, Connon CJ, Dennis S, Khan S. Transparency, swelling and scarring in the corneal stroma. Eye. 2003;17: 927-936.

8. Hart RW, Farrell RA. Light scattering in the cornea. J Opt Soc Am. 1969;59:766-774.

9. Maurice DM. The structure and transparency of the cornea. $J$ Physiol. 1957;136:263-286.

10. Aghamohammadzadeh H, Newton RH, Meek KM. X-ray scattering used to map the preferred collagen orientation in the human cornea and limbus. Structure. 2004;12:249-256.

11. Boote C, Dennis S, Meek KM. Spatial mapping of collagen fibril organisation in primate cornea- an X-ray diffraction investigation. $J$ Structural Biol. 2004;146:359-367.

12. Hayes S, Boote $\mathrm{C}$, Lewis $\mathrm{J}$, et al. Comparative study of fibrillar collagen arrangement in the corneas of primates and other mammals. Anat Rec. 2007;290:1542-1550.

13. Safranski TJ, Lamberson WR, Keisler DH. Correlations among 3 measures of puberty in mice and relationships with estradiol concentration and ovulation. Biol Reprod. 1993;48:669-673.

14. Fullwood NJ, Meek KM. An ultrastructural, time-resolved study of freezing in the corneal stroma. J Mol Biol. 1994;236:749-758.

15. Boote C, Dennis S, Newton RH, Puri H, Meek KM. Collagen fibrils appear more closely packed in the prepupilliary cornea-optical and biomechanical implications. Invest Ophthalmol Vis Sci. 2003; 44:2941-2948.

16. Meek KM, Quantock AJ. The use of X-ray scattering techniques to determine corneal ultrastructure. Prog Retinal Eye Res. 2001;20: 95-137.

17. Abahussin M, Hayes S, Knox Cartwright N, et al. 3D collagen orientation study in human cornea using X-ray diffraction and femtosecond laser technology. Invest Ophthalmol Vis Sci. 2009; 50:5159-5164.

18. Daxer A, Fratzl P. Collagen fibril orientation in the human corneal stroma and its implications in keratoconus. Invest Ophthalmol Vis Sci. 1997;38:121-129.

19. Beecher N, Chakravarti S, Joyce S, Meek K, Quantock AJ. Neonatal development of the corneal stroma in wild-type and lumican-null mice. Invest Ophthalmol Vis Sci. 2006;47:146-150.

20. Boote $\mathrm{C}$, Hayes $\mathrm{S}$, Jones $\mathrm{S}$, et al. Collagen organization in the chicken cornea and structural alterations in the retinopathy, globe enlarged (rge) phenotype - an X-ray diffraction study.J Struct Biol. 2008;161:1-8.

21. Maurice DM. The cornea and sclera. In: Davson $\mathrm{H}$, ed. The Eye. New York: Academic Press; 1969;489-599.

22. Graham B, Judge SJ. Normal development of refractive state and ocular component dimensions in the marmoset (Callithrix jacchus). Vis Res. 1999;39:177-187.

23. Howlett MHC, McFadden SA. Emmetropization and schematic eye models in developing pigmented guinea pigs. Vis Res. 2007;47: $1178-1190$. 
24. Mutti DO, Mitchell GL, Jones LA, et al. Axial growth and changes in lenticular and corneal power during emmetropization in infants. Invest Ophthalmol Vis Sci. 2005;46:3074-3080.

25. Schmucker C, Schaeffel F. A paraxial schematic eye model for the growing C57BL/6 mouse. Vis Res. 2004;44:1857-1867.

26. Troilo D, Judge SJ. Ocular development and visual deprivation myopia in the common marmoset (Callithrix jacchus). Vis Res. 1993;33:1311-1324.

27. Wallman J, Adams JI, Trachtman JN. The eyes of young chickens grow toward emmetropia. Invest Opbthalmol Vis Sci. 1981;20: 557-561.
28. York MA, Mandell RB. A new calibration system for photokeratoscopy, 2: corneal contour measurements. Am J Optom. 1969;46: $818-825$

29. Inagaki Y. The rapid change of corneal curvature in the neonatal period and infancy. Arch Opbthalmol. 1986;104:1026-1027.

30. Smith E. Environmentally induced refractive errors in animals. In: Rosenfield M, Gilmartin B, eds. Myopia and Near Work. Oxford: Butterworth Heinemann; 1998:57-90.

31. Zhou XT, Shen MX, Xie J, et al. The development of the refractive status and ocular growth in C57BL/6 mice. Invest Ophthalmol Vis Sci. 2008;49:5208-5214. 